\title{
TANNAKA-KREIN DUALITY FOR COMPACT GROUPOIDS II, DUALITY
}

\author{
Massoud AMINI
}

Abstract. We show that from the representation theory of a locally non-trivial compact groupoid whose irreducible representations separate its points, one can reconstruct the groupoid using a procedure similar to the Tannaka-Krein duality for compact groups. We study the Fourier and Fourier-Plancherel transforms and prove the Plancherel theorem for compact groupoids.

Mathematics subject classification (2010): Primary 43A65, Secondary 43A40.

Keywords and phrases: Topological groupoid, representations, Fourier transform, central elements, Plancherel theorem, Tannaka groupoid.

\section{REFERENCES}

[1] Massoud Amini, Tannaka-Krein duality for compact groupoids I, representation theory, Advances in Mathematics, 214 (2007), 78-91.

[2] R. D. Bos, Groupoids in Geometric Quantization, Ph.D. Thesis, Universiteit Nijmegen, 2007.

[3] Ronald Brown, Topology and Groupoids, BookSurge, LLC, Charleston, SC, 2006.

[4] Charles Ehresmann, Catégories topologiques et catégories différentiables, Colloque Géom. Diff. Globale, Bruxelles, 1958, 137-150.

[5] A. Joyal, R. Street, An introduction to Tannaka duality and quantum groups, in Category theory, Proceedings of the international conference, Como, 1990, A. Carboni et al (eds.) Lecture Notes in Mathematics 1488, Springer Verlag, Berlin, 1991.

[6] Kirill C. H. MackenzIE, General Theory of Lie Groupoids and Lie Algebroids, London Mathematical Society Lecture Note Series 213, Cambridge University Press, Cambridge, 2005.

[7] Alan L. T. PAterson, Groupoids, inverse semigroups, and their Operator Algebras, Progress in Mathematics 170, Birkh ä user, Boston, 1999.

[8] JeAn Renault, A groupoid approach to $C^{*}$-algebras, Lecture Notes in Mathematics 793, SpringerVerlag, 1980.

[9] JEAn Renault, Représentation des produits croisés d'algébres de groupoides, J. Operator Theory, 18, 1 (1987), 67-97.

[10] A. K. SEDA, Haar measures for groupoids, Proc. Roy. Irish Acad. Sect. A, 76, 5 (1976), 25-36.

[11] Alan Weinstein, Groupoids: unifying internal and external symmetry. A tour through some examples, Notices Amer. Math. Soc., 43, 7 (1996), 744-752. 